\title{
Tranversalización de la Perspectiva de Género: Una mirada crítica a los programas de estudio de la Facultad de Ciencias Sociales. ¿Cuánto hemos avanzado?
}

Transversality of the Gender Perspective: A Critical Look at the Study Programs of the Faculty of Social Sciences. How Far Have We Come?

\author{
Nalda Arias-Cascante \\ Universidad Nacional \\ Escuela de Administración \\ Heredia, Costa Rica \\ narias@una.cr \\ Ana Lucía Bustos-Vásquez \\ Universidad Nacional \\ Escuela de Sociología \\ lucia_bustos@yahoo.com \\ Celia Viquez-Rojas \\ Universidad Nacional \\ Escuela de Psicología \\ cevispic@yahoo.com.mx
}

Recibido: 02/10/2014 Aceptado: 27/06/2016

Resumen: Este trabajo se corresponde con el proyecto: "Análisis de Transversalización de Género en la Facultad de Ciencias Sociales". Nuestro objetivo se focaliza en indagar ¿cómo se ha transversalizado la perspectiva de género en las carreras de la Facultad C.S.? Esta propuesta acoge como fundamento político-institucional la normativa de la Universidad Nacional, que apuesta por la inclusión del género y la equidad como ejes transversales para asumirse en los ámbitos del quehacer universitario. Como fundamento teóricometodológico se presenta una investigación desde unas miradas de mujeres académicas en la academia, atendiendo al análisis de dos dimensiones: (1) género como eje transversal y (2) como visibilización de mujeres en la academia. Las evidencias dilucidan limitaciones con respecto a la transversalización en términos efectivos. Se sigue proyectando roles estereotipados en la docencia, 
Revista Universidad en Diálogo • Vol. 6, N. 2 2, Julio-diciembre 2016, pp. 123-144

ISSN 2215-2849 • EISSN: 2215-4752

DOI: http://dx.doi.org/10.15359/udre.6-2.7

y profesionalización segregada por sexo. Esto es un reto que debe asumir la academia para responder a las dinámicas de desigualdad por las que aún transitan las mujeres universitarias.

Palabras clave: perspectiva de género, transversalización, mujeres y academia.

\begin{abstract}
This work corresponds to the project: "Analysis of Gender Mainstreaming in the Faculty of Social Sciences". Our goal focuses on investigating how has implemented the gender perspective in the careers of the Faculty of Social Sciences? This proposal as the foundation welcomes political-institutional regulations of the National University, that bet by the inclusion of gender and equity as transverse axis to be assumed in the areas of academic. As a theoretical basis-methodological presents a research from some glances of academic women in the academy, based on the analysis of two dimensions: (1) gender as a transverse axis and (2) such as visibility of women in the academy. Evidence elucidates limitations with regard to mainstreaming in effective terms. Is still projecting stereotyped roles in teaching, and professionalization segregated by sex. This is a challenge that must be addressed the academy to respond to the dynamics of inequality that still by the transit of university women.
\end{abstract}

Keywords: gender perspective, mainstreaming, women and academy.

\title{
Valoraciones introductorias
}

La historia de la humanidad ha estado marcada fundamentalmente por un orden androcéntrico de producción y visión de mundo que ha discriminado a las mujeres, a la población infantil, a poblaciones con rasgos y características étnico-culturales que se han considerado inferiores.

Para el caso que nos ocupa, centramos esta propuesta en el análisis de la discriminación y las desigualdades que las mujeres han sufrido producto de una sociedad patriarcal. No pocos casos conocemos en la historia y en la mitología sobre esa discriminación; desde la construcción simbólica de la mujer virgen o prostituta que se materializa en los discursos, en este particular, judeo-cristianos, como por ejemplo, en la imagen idealizada de la virgen María y su contraposición María Magdalena. Así se evidencia que la presencia de las mujeres en la historia ha sido asumida desde idealizaciones de bondad y perfección hasta la encarnación de la maldad, llegando incluso a la concreción de todo tipo de violencias, recordando para tal fin, la quema de brujas (mujeres sabias que en general desafiaban el poder y las normas de una lógica androcéntrica), la lapidación, infibulación y escisión como prácticas culturales que aún, hoy en día, son llevadas a cabo como ritos en las sociedades. 
A pesar de la posición que las mujeres han tenido bajo lógicas de discriminación en una sociedad patriarcal, han generado estrategias reivindicativas de posicionamiento político, económico, social, cultural y científico, que les confiere avances en materia de derechos humanos. Ver recuadro 1.

Como parte de los procesos de reivindicación y de mitigación contra las desigualdades, hay que rescatar el trabajo realizado desde los movimientos feministas y los grupos organizados de mujeres, cuya resonancia ha tenido un impacto significativo en el marco del derecho internacional y la institucionalización de las propuestas feministas en el Estado y especialmente en el ámbito de la Educación Superior.

Recuadro 1: Mujeres Escribas: Tejedoras de pensamiento.

"Durante siglos la misoginia y el racismo, justificaron la exclusión de las mujeres, de las aulas y la ciencia. La educación llegó a considerarse un espacio exclusivamente masculino y de élites, generando asimetrías que apenas empiezan a remontarse hacia finales del siglo XIX cuando algunas pioneras flanquearon las puertas de los recintos universitarios".

(Extracto, Ana Silvia Monzón, Guatemala, 2011).

Se debe mencionar que a nivel internacional en el marco de la Plataforma de Acción de la IV Conferencia Mundial sobre la Mujer, que establece la estrategia de política de igualdad entre mujeres y hombres, mainstreaming de género (Beijing, 1995) o transversalidad de género, que implica una revisión crítica acerca de quiénes elaboran las políticas, programas institucionales, y quiénes toman las decisiones y qué efectos diferenciados conllevan en las condiciones de vida de las mujeres y los hombres, para impactar en la reducción de brechas de género.

Costa Rica ha suscrito y ratificado los acuerdos de esta Conferencia, así como muchos de los acuerdos internacionales de la Organización de las Naciones Unidas así como la Convención Contra toda Forma de Discriminación contra la Mujer (CEDAW, por sus siglas en inglés). El país es Estado Parte del Protocolo Opcional de esta Convención -ratificado el 20 de setiembre del 2009- y de la Convención de Belem de Pará sobre la erradicación y eliminación de toda violencia contra las mujeres, entre otros cuerpos jurídicos internacionales. En consecuencia, el país aprobó la Política Nacional para la Igualdad y Equidad de Género, PIEG 2007-2017, en el año 2007; normativa que se constituye en un gran logro porque define, orienta y direcciona la gestión en materia de igualdad y equidad para las mujeres costarricenses. 
Revista Universidad en Diálogo • Vol. 6, N. 2 2, Julio-diciembre 2016, pp. 123-144

ISSN 2215-2849 • EISSN: 2215-4752

DOI: http://dx.doi.org/10.15359/udre.6-2.7

La Universidad Nacional desde sus reflexiones internas, ha fundamentado una normativa institucional vinculada con el marco jurídico internacional en materia de género. El Plan Global Institucional 2004- 2011, presenta el género como uno de los valores que integran el quehacer universitario, además considera los acuerdos del III Congreso Universitario (2001) donde se aprueba el género y la equidad como dos de los ejes transversales.

El 21 de mayo del 2010 se aprueba la Política de Igualdad y Equidad de Género (PIEG-UNA), como un instrumento jurídico que fundamenta la temática de la equidad en este centro de estudios superiores. La aprobación de la PIEGUNA da sustento a este nuevo régimen de interrelaciones para guiar los asuntos de la igualdad y equidad en la institución y, a partir de ello regir así la vida de las relaciones entre las mujeres y los hombres, analizando y redefiniendo los aspectos de poder, jerarquía y socialización, bajo lógicas de simetría y justicia redistributiva de las relaciones humanas. Este cuerpo normativo conlleva primero un profundo análisis y valoración de las necesidades en esta temática y, posteriormente, la toma de decisiones que orientarán la gestión.

La Política plantea elementos relacionados con la promoción de la participación paritaria de mujeres y hombres en los órganos de decisión, garantizando el acceso y desarrollo de la comunidad universitaria en condiciones de igualdad. Propicia el uso de un lenguaje inclusivo en todas las formas de comunicación interna y en las relaciones externas de la Universidad. Veamos cómo se expresan de forma concretan estas valoraciones:

La Universidad Nacional comprometida con la igualdad de oportunidades sin distingo de ningún tipo y en consonancia con sus propósitos estratégicos, es decir, ser una Universidad Humanista, Innovadora y con Compromiso Social, formula las presentes políticas para la igualdad y la equidad de género. (GACETA $\mathrm{N}^{\circ}$ 09-2010, 15 de junio, 2010, p. 4)

Basadas en el fundamento anterior, se hace necesario reflexionar con respecto a los alcances y limitaciones de la Política de Igualdad y Equidad de Género (PIEG-UNA) en la Facultad de Ciencias Sociales. El objetivo de esta ponencia es presentar un análisis con respecto a la incorporación de la transversalización de género en la currícula de estudios de las unidades académicas de la Facultad de Ciencias Sociales. Nuestro principal cuestionamiento es ¿Cómo se ha transversalizado la perspectiva de género (P.G.) en relación con la visibilización de las mujeres en la Facultad de Ciencias Sociales? ¿Pero, qué entendemos por transversalización de género en la educación superior? 
Los estudios desde la perspectiva de género han ahondado en la identificación de las desigualdades que históricamente han experimentado las mujeres. Si bien las instituciones de educación superior son espacios de promoción de los derechos de las personas, no han escapado de la reproducción de estereotipos que perpetúan las condiciones asimétricas entre mujeres y hombres. En este contexto transitamos.

La perspectiva de género es una herramienta que permite observar la realidad, implica una mirada más profunda, proyectando el carácter de una perspectiva teórica y transdisciplinar que se enriquece con la visibilización de referentes metodológicos abiertos hacia comprensiones diversas y complejas de la realidad social. Lo que le constituye además en un referente para la construcción y reconocimiento de saberes que han tenido cabida tímida en la academia. En particular, permite identificar la desigualdad existente en la diferenciación de los roles y actividades que llevan a cabo las mujeres y los hombres en una sociedad (reconociendo clase y etnia), así como observar asimetrías, relaciones de poder e inequidades.

El enfoque de género permite analizar las causas que producen las desigualdades y definir mecanismos para superar las brechas. Plantea como uno de sus principales objetivos colaborar en la construcción social y resignificación de la historia, la sociedad, la cultura y la política. En la academia, constituye el compromiso a nivel institucional dirigido a fomentar cambios sustantivos en la condición y posición de las mujeres con mayor equidad, solidaridad y justicia, aplicable a todos los ámbitos de la vida universitaria que trasciende a los otros ámbitos de la vida por los que transitan las personas que tiene y han tenido la posibilidad de caminar por las aulas universitarias.

Es por ello que se define la perspectiva de género como un medio para mitigar las condiciones de desigualdad y se le apuesta a la transversalización como estrategia para incorporar de forma sistemática las preocupaciones, experiencias, necesidades prácticas e intereses estratégicos diferenciados entre mujeres y hombres, en la dinámica universitaria, con el fin de promover la equidad de género. ${ }^{1}$

¿Qué vamos a transversalizar y en qué ámbitos de acción? La transversalización de la perspectiva de género debe permear los espacios de toma de decisión e influir positivamente, en una mayor participación de las mujeres en condiciones de igualdad y equidad. En este sentido, resulta viable

Elaboración propia basada en la revisión de material bibliográfico. Considerando las siguientes autoras: Fainholc, Beatriz, 2011; Monzón, Silvia, 2009; Beltrán, Elena, 2008, Lagarde, Marcela, 1996. 
el trabajo equitativo en la generación de las políticas, en los espacios de trabajo, y en la dirección y desarrollo (planificación, ejecución, evaluación y seguimiento) de la vida académica, especialmente en la Facultad de Ciencias Sociales. Entonces, la transversalización, requiere de acciones, estrategias y programas específicos, de una agenda institucional que promueva y brinde recursos concretos, procesos de capacitación y realice las reformas necesarias para incorporar perspectiva de género progresivamente, en los diversos espacios académico-institucionales (profesional académico-administrativo-estudiantado).

Este proceso de transversalización debe trabajarse, al menos, en tres niveles o ámbitos de acción: el político-institucional (en nuestro caso hacemos referencia al marco normativa de la UNA) con políticas afirmativas que puedan generar avance en la eliminación de la segregación disciplinaria en las carreras, -esto como una proyección-, lo que debe decantar en un efecto directo en el nivel de formación del estudiantado, incluyendo como eje transversal la perspectiva de género, y por lo tanto, figurar en la propuesta curricular de cada unidad académica, es decir, que se vincule directamente en la estructuración de los planes de estudio, y específicamente en los programas de cursos, como referentes básicos en formación universitaria.

¿Cómo abordamos en términos metodológicos este trabajo? En esta oportunidad revisamos aspectos sobre los procesos de transversalización de la perspectiva de género en los programas de los cursos que se imparten en las diferentes carreras de las unidades académicas de la Facultad de Ciencias Sociales. ¿Cómo lo hacemos?

Como primer momento, en tanto estrategia metodológica, realizamos una revisión documental de la PIEG-UNA y el Modelo Pedagógico de la Universidad Nacional y hacemos uso de una serie de material bibliográfico referido a teoría de género, teoría feminista e investigaciones en el ámbito académico que tienen como fundamento el enfoque de género. Ello nos permite definir y dar sustento a los conceptos que facilitan el diseño de un marco referencial para el análisis de nuestra temática investigativa, que centraliza nuestra atención en dos dimensiones: (1) Particularidades (elementos, ideas, valores) referidas a la perspectivas de género en los programas de cursos de las unidades académicas de la Facultad de Ciencias Sociales, concretamente en elementos de equidad y respeto a la diversidad y derechos humanos. (2) Participación de mujeres en la academia referido con elementos de visibilización y presencia de mujeres en los programas y planes de estudio de las carreras de la Facultad. 
Para dar sustento y poder contrastar con nuestro contexto de la praxis, las unidades académicas de la Facultad, se constituyen en nuestro referente empírico. Como segundo momento, planteamos el estudio bajo un enfoque cuantitativo que asume como población a la totalidad de las unidades académicas. Realizamos, en términos absolutos, una revisión de 318 programas que represente más de $95 \%$ de la totalidad de la malla curricular ofertadas por las carreras de bachillerato y licenciatura en: Historia, Relaciones Internacionales, Sociología, Psicología, Administración de Empresas, Economía, Planificación Económico Social y Secretariado Profesional. Los programas de los cursos corresponden al primer y segundo ciclo de años comprendidos entre el 2010-2014 y han sido suministrados por cada una de las Unidades Académicas. Ver tabla 1.

\section{Tabla 1}

Escuelas de la Facultad de Ciencias Sociales y cantidad de programas analizados $(\boldsymbol{n}=318)$

\begin{tabular}{lclc}
\hline \multicolumn{1}{c}{ Escuelas/ Carrera } & $\mathbf{n}=$ & Escuelas/ Carrera & $\mathbf{n}=$ \\
\hline Secretariado Profesional & $\mathbf{4 5}$ & Relaciones Internacionales & $\mathbf{4 3}$ \\
Psicología & $\mathbf{3 8}$ & Planificación & $\mathbf{3 8}$ \\
Historia & $\mathbf{3 4}$ & Economía & $\mathbf{4 6}$ \\
\hline Sociología & $\mathbf{2 8}$ & Administración & $\mathbf{4 5}$ \\
\hline
\end{tabular}

$\mathbf{n}=$ Corresponde a la cantidad de programas procesados en cada una de las carreras que se imparten en la Facultad de Ciencias Sociales. UNA.

Como criterios de selección tomamos en consideración la vigencia del plan de estudios de cada una de las carreras de la Facultad. Se excluyen programas de cursos optativos y sólo se analizan programas en los que aparecen como titulares del curso, personas que laboran en la institución al momento de desarrollarse el estudio. Para las carreras de Relaciones Internacionales y Administración se selecciona, a nivel de licenciatura, el énfasis que dio origen a la carrera. 
¿Cómo y con cuáles herramientas metodológicas observamos? Nuestras unidades de análisis se materializan en los programas de los cursos y su contenido especifico en materia de perspectiva de género. Para el desarrollo del estudio de los programas hacemos uso del análisis contenido, con el fin de realizar inferencias y describir tendencias de lo escrito en correspondencia con ideas, valoraciones, criterios discursivos en relación con: a) qué se dice, b) cuándo, c) dónde y por qué, d) quién registra lo que se describe en el programa de un curso, en las diferentes carreras de la Facultad de Ciencias Sociales.

La presencia de términos o vocablos referidos a palabras claves como género, derechos humanos, diversidades; el uso de artículos (la / el / lo) hasta la utilización de lenguaje inclusivo, la presencia o no de material bibliográfico elaborado por mujeres y/u hombres son relevantes para el análisis. Más allá de las palabras, vocablos, temas o categorías en términos de cantidad, lo destacable para esta investigación es el significado que expresan, cómo se presentan estas palabras en el documento, la frecuencia en la que puedan aparecer en el texto del programa, ya que es a partir de esas manifestaciones lingüísticas que podemos formular inferencias sobre significaciones no lingüisticas, asumiendo miradas epistemológicas desde la perspectiva de género. Posteriormente se trasladan el análisis de corte cualitativo a una base de datos con registros numéricos para obtener frecuencias relativas con respecto a presencia de mujeres en la academia y se utilizan como códigos según una escala numérica la evidencia o no evidencia, la incorporación o no incorporación, el registro o no registro de la materialización de los vocablos o ideas indicadas en párrafos anteriores.

¿Qué observamos, qué se transversaliza? El uso del lenguaje, la presencia o no de mujeres docentes, la incorporación de material bibliográfico elaborado por mujeres y estrategias pedagógicas que tiendan hacia procesos participativas y/o valores que expresan derechos humanos, procesos género sensitivos, de equidad y respeto hacia la diversidad, constituyen las primeras dimensiones de análisis que estudiamos en esta investigación, y las hemos definido como subdimensiones que nos permiten, de forma precisa, reconocer el avance, la incorporación de la perspectiva de género en los procesos de formación de nuestra Facultad.

Dichas valoraciones son el soporte de nuestra reflexión teórico-conceptual que dio origen al proceso de operacionalización de dimensiones y sub-dimensiones para el análisis que se encuentran en la tabla 2. 
Tabla 2

Formas de "medición” en relación con las subdimensiones de análisis y escalas de medición utilizadas

\begin{tabular}{|c|c|c|c|}
\hline \multicolumn{2}{|c|}{ Definición operacional } & \multirow{2}{*}{$\begin{array}{c}\text { Valoración específica } \\
\text { para analizar }\end{array}$} & Categorización \\
\hline Dimensión & Subdimensión & & \multirow{2}{*}{$\begin{array}{l}\text { 1. Se registra una mujer } \\
\text { 2. Se registra un hombre. } \\
\text { 3. Registra una mujer y } \\
\text { un hombre } \\
\text { 9. No registra quien } \\
\text { asume el curso. }\end{array}$} \\
\hline \multirow{4}{*}{ 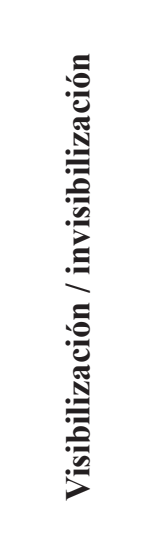 } & \multirow[t]{3}{*}{$\begin{array}{l}\text { Presencia de } \\
\text { mujeres }\end{array}$} & $\begin{array}{l}\text { Asignación de cursos } \\
\text { según sexo }\end{array}$ & \\
\hline & & $\begin{array}{l}\text { Visibilización de las } \\
\text { mujeres en alguna } \\
\text { parte del programa }\end{array}$ & \multirow{6}{*}{$\begin{array}{l}\text { 1. Se evidencia en el } \\
\text { texto } \\
\text { 2. Se evidencia en parte } \\
\text { del texto. } \\
\text { 3. No se evidencia en el } \\
\text { texto }\end{array}$} \\
\hline & & $\begin{array}{l}\text { Presentación de } \\
\text { material didáctico de } \\
\text { creación propia }\end{array}$ & \\
\hline & $\begin{array}{l}\text { Uso del } \\
\text { lenguaje }\end{array}$ & $\begin{array}{l}\text { Uso de lenguaje } \\
\text { inclusivo }\end{array}$ & \\
\hline \multirow{3}{*}{ 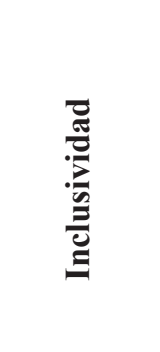 } & \multirow{3}{*}{$\begin{array}{l}\text { Propuestas } \\
\text { pedagógicas } \\
\text { inclusivas }\end{array}$} & Acciones afirmativas & \\
\hline & & $\begin{array}{l}\text { Evidencia/presencia } \\
\text { de derechos humanos }\end{array}$ & \\
\hline & & $\begin{array}{l}\text { Actividades } \\
\text { pedagógicas que } \\
\text { incluyan el derecho de } \\
\text { las personas }\end{array}$ & \\
\hline
\end{tabular}

\section{¿Cuáles son nuestros hallazgos? ¿Cómo se ha transversalizado la perspectiva de género en relación con la visibilización de las mujeres en la Facultad de Ciencias Sociales?}

Partiendo de nuestras dimensiones de análisis, presentaremos los hallazgos en relación con el uso del lenguaje, presencia o no de mujeres docentes, incorporación de material bibliográfico elaborado por mujeres y estrategias pedagógicas. 
Aunque la formalización académica de la perspectiva y los estudios de género deconstruyen los marcos disciplinares de los estudios tradicionales (Historia, Economía, Literatura, Sociología, Psicología, etc.) para rebatir visiones de objetividad científica como garantía de imparcialidad y se han realizado importantes aportes, reconociendo epistemologías del posicionamiento o conocimiento situado (Haraway, 1995) presente en las más avanzadas propuestas teóricas feministas|, aún hoy en el sistema educativo abunda una estereotipia de la forma de organizar las materias y los contenidos que se enseñan, en los libros y materiales didácticos y las prácticas evaluativas que esperan determinados logros para los estudiantes y las estudiantes. En fin podemos evidenciar en las lógicas formales de la educación mecanismos que refuerzan condiciones de desigualdad (Fainholc, 2011).

En particular cuando realizamos un análisis somero con respecto a unidades académicas específicas del área de las Ciencias Sociales, podemos avizorar que los avances en materia de incorporación y transversalización de la perspectiva de género han sido muy limitados y aunque se profesa "el género" como un eje en términos de normativa que hay que incorporar, todavía no se pueden evidenciar muchos avances.

\section{Asignación de cursos por sexo}

Mujeres y varones han transitado y transitan por las aulas universitarias y se han ido derribando valoraciones estereotipadas con respecto a la valía y la presencia de mujeres en espacios desde los que, según una visión de mundo androcéntrico, eran una posibilidad sólo para los varones. Sin embargo una muestra de las disparidades, incluso a niveles cuantitativos, que podemos observar es la cantidad de hombres y mujeres que tienen un espacio en la vida académica de la Facultad de Ciencias Sociales (F.C.S). En este particular, podemos evidenciar que de un total de 289 académicas y académicos que laboran en la Facultad, 95 mujeres y 194 hombres (Registro-UNA, 2014).

Cuando revisamos los programas que se imparten en las diferentes carreras de las unidades académicas de la F.C.S, solo $37,7 \%$ de los cursos se han adjudicado a las académicas que forman parte del cuerpo docente. (Ver tabla 3 ).

Las preguntas claves que aquí colocamos nos hacen pensar en que la presencia y paso de muchas mujeres por las aulas universitarias no se corresponden o no tiene un fiel reflejo cuando se trata de realizar contrataciones o encontrarlas ejerciendo su profesionalización en los centros de educación superior. ¿Cuáles son las labores que desempeñan las mujeres en la 
academia? Sin desmerecer sus aportes en áreas de gestión administrativa ¿será que los espacios donde se asume una profesional en los claustros educativos aún está lejana como académica/docente, en tanto haya mayor distancia de las etapas iniciales del sistema educativo formal (enseñanza en el nivel de prescolar y de educación general básica)?

\section{Tabla 3}

Asignación de cursos según sexo de la persona que lo facilita. Facultad de Ciencias Sociales $n=318$

\begin{tabular}{lcc}
\hline ¿Quién facilita el curso? & Valores absolutos & $\begin{array}{c}\text { Valores relativos } \\
(\%)\end{array}$ \\
\hline Se asigna a una mujer & 120 & 37,7 \\
Se asigna a un varón & 156 & 49,1 \\
Se asigna a una mujer y a un hombre & 18 & 5,7 \\
No indica asignación & 24 & 7,5 \\
\hline \multicolumn{1}{c}{ Total } & $\mathbf{3 1 8}$ & $\mathbf{1 0 0 , 0}$ \\
\hline
\end{tabular}

Si ponemos en consideración las disciplinas en las que se proyecta mayor o menor presencia de mujeres en la academia, podemos precisar que existe una tendencia de mayor registro o asignación de programas a profesores en unidades académicas como: Relaciones Internacionales, Administración y Planificación Económico Social, disciplinas que pudiéramos asociar con asignaciones estereotipadas. No podemos afirmar esta valoración para el caso de la carrera de Economía, ya que en este análisis más de la mitad de los programas de los cursos consultados no registran quién los imparte. Sin embargo, para el primer ciclo lectivo del 2014, se contó con 30 profesores y solo 6 profesoras que imparten cursos (Registro-UNA, 2014). Lo que refuerza la idea de disparidad en tanto presencia de mujeres según división sexual del trabajo, en este caso de forma específica en las labores docentes.

Mayor presencia de cursos asignados a mujeres está registrada en unidades académicas de Secretariado Profesional, Psicología, Historia y Sociología (figura 1). Nuevamente asignaciones que tienen fiel correspondencia con una división sexual del trabajo, siguiendo la visión hegemónica y dicotómica que perpetúa condiciones de desigualdad. 


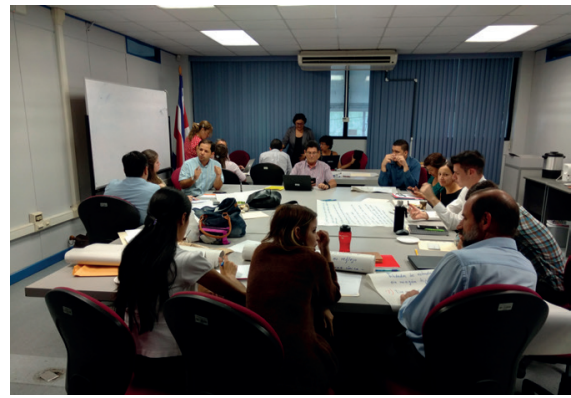

Mayor asignación de cursos a varones: Relaciones Internacionales, Administración, Planificación Económica y Social.

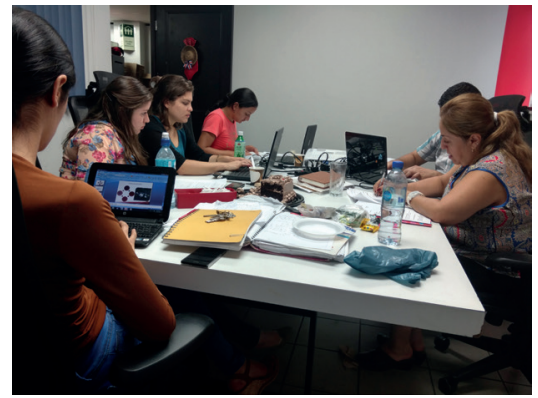

Mayor asignación de cursos a mujeres:

Secretariado, Profesional, Psicología, Historia, Sociología.

Figura 1. Presencia de mujeres y varones según registro de facilitación de cursos*.

Nota: Análisis de 318 programas de las diferenes unidades académicas. Facultad de Ciencias Sociales. UNA.

\section{Autoras en las bibliografías de los programas analizados}

Un cúmulo de aportes y desarrollos teórico-metodológicos elaborado tanto por mujeres como por varones pueden encontrarse en las diferentes disciplinas desde las que se ha validado el conocimiento. Así podemos identificar un sin número de material documental, audiovisual de corte investigativo y formativo disponible hoy, incluso haciendo "uso de solo un clic" en nuestras computadoras. Aproximadamente más de 13,100 resultados fueron desplegados en un computador al colocar como descriptor en el buscador de google académico "documentos e investigaciones escritas por mujeres en América Latina"; no obstante aún la presencia de las mujeres y la visibilización de sus aportes a la academia es limitado también en este sentido.

Del total de los programas analizados sólo cerca de $20 \%$ presenta documentos con autoría de mujeres, no obstante debemos indicar que esta presencia no supera más de 10 documentos en una bibliografía que, por ejemplo, reporte más de 20 documentos que deben ser estudiados por las personas que participan en los cursos. Ver figura 2.

El nulo registro de material con autoría de académicas o investigadoras en el área de las Ciencias Sociales se evidencia en $40 \%$ de los programas analizados.

\footnotetext{
El ejercicio de búsqueda de material bibliográfico elaborado por mujeres en América Latina, se realiza en agosto de 2014 y este es solo un ejemplo de la cantidad de material disponible que puede ser utilizado en las diferentes disciplinas de las Ciencias Sociales.
} 


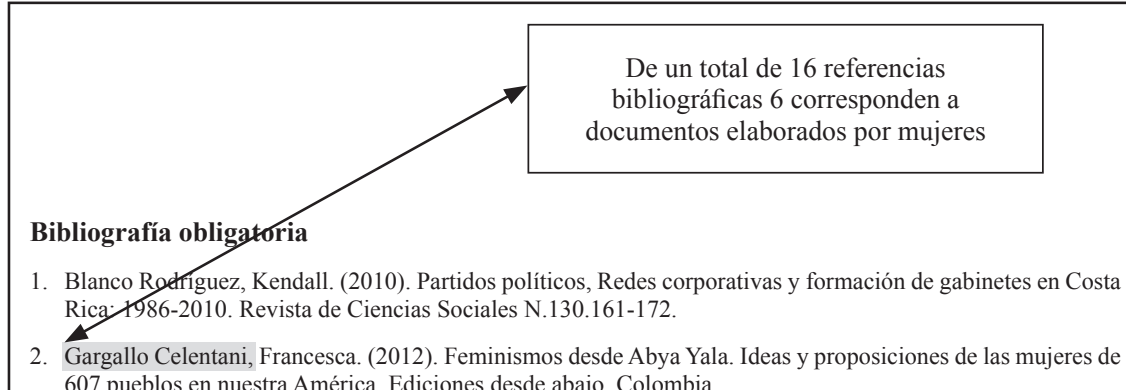
607 pueblos en nuestra América. Ediciones desde abajo. Colombia.

3. Gasper, Des. (2011). El enfoque de seguridad humana como marco para la ética del cambio ambiental global disponible en http://www.mundosigloxxi.ciecas.ipn.mx/pdf/v06/23/01.pdf. Revista mundo siglo XXI. Número 23, primavera 2010-2011.

4. Girola, Lidia. (2007). Imaginarios socioculturales de la modernidad. Aportaciones recientes y dimensiones del análisis para la construcción de una agenda de investigación. Sociología, año 22, número 64, mayo-agosto de 2007, pp 45-76 disponible en http://www.revistasociológica.com.mx/pdf/6403.pdf el 11 de enero de 2013.

5. Hernández, Juan. (s.f). La era de la información ¿En qué mundo estamos viviendo. Revista ENVIO, Revista mensual de análisis en Nicaragua y Centroamérica, Universidad Centroamericana UCA, Nicaragua. (41 páginas). Disponible en www.envio.org.ni/articulo/1222 el 11 de enero de 2013.

6. Ianni, Octavio. (2005). La sociología y el mundo moderno. XXI Editores, México. Páginas 9-32,45-65,128$138,150-157$ y $168-208$.

7. Kostas Vergopoulus. (2011). La emergencia del nuevo poder financiero http://www.mundosigloxxi.ciecas. ipn.mx/pdf/v06/24/02.pdf. Revista mundo siglo XXI. Número 24,2011. Disponible el 15 de enero de 2013.

8. Melucci, Alberto. (1989). El tiempo de la diferencia: condición femenina y movimientos de las mujeres. Revista Sociológica. Año 4 No. 10 disponible en http://www.revistasociológica.com.mx/pdf/1012.pdf el 15 de enero de 2013.

9. Olivera, Margarita. (2007). Un marco de análisis sociológico de los miedos modernos: vejez, enfermedad y muerte. Sociológica, año 22, número 64, mayo-agosto de 2007, pp. 119-149 disponible en http://www.revistasociológica.com.mx/pdf/6405.pdf el 11 de enero de 2013.

10. Beck, Ulrich. (1998). La sociedad del riesgo. Paidós. España.

11. Rivera Cusicanqui, Silvia. (2010). Violencia (re) encubiertas en Bolivia. Editorial Piedra Rota. La Paz. Bolivia.

12. Sagot, Monserrat. (2007). (Re) Definiendo las identidades y acción política: multitudes diversas, sujetos colectivos y movimientos sociales en Centroamérica del nuevo milenio. Cuadernos de Sociología. No. 7. Facultad de Ciencias Sociales. Universidad de Costa Rica. Costa Rica.

13. Sánchez Albarrán, Armando. (2012). Sociología Rural: nueva agenda de investigación, viejos problemas sin solución. Globalización y agricultura. Nuevas perspectivas en sociología rural. UAAAN-UAdeC.

14. Sangría Obediente, Mario. (2011). Del capitalismo al socialismo del Siglo XXI. Perspectiva desde antropología crítica. BCV. Caracas, Venezuela. (III Parte, Capítulo 16. El neoevolucionismo y la energía: legitimación ideológica del neocolonialismo).

15. Tamayo, Sergio. (1995). Movimientos sociales modernos, revueltas o movimientos antisistémico. Revista Sociológica. Año 10 No. 28 disponible en http://www.revistasociológica.com.mx/pdf/2813.pdf el 15 de enero de 2013.

16. Zabludovsky Kuper, Gina. (2007). Sociología y Cambio Conceptual. Siglo XXI Editores, México, D.F.

Figura 2. Bibliografía en programa de curso. 
Los materiales que abundan en las bibliografias de los programas de los cursos tienen autoría masculina, ello sin ahondar en que este tipo de material documental puede afianzar propuestas clásicas de un sistema mundo patriarcal y androcéntrico, que tiende a opacar los aportes de la teoría feminista y sus consecuentes valoraciones como enfoque o perspectiva de género (tabla 4).

Tabla 4

Presencia de Autoras/investigadoras en la bibliografia. Facultad de Ciencias Sociales $n=318$

\begin{tabular}{|c|c|c|}
\hline $\begin{array}{l}\text { ¿Se incorpora material elaborado por } \\
\text { mujeres en la bibliografia del programa? }\end{array}$ & $\begin{array}{l}\text { Valores } \\
\text { absolutos }\end{array}$ & $\begin{array}{c}\text { Valores relativos } \\
(\%)\end{array}$ \\
\hline Se incorpora & 63 & 19,8 \\
\hline Se incorpora en parte & 101 & 31,8 \\
\hline No se incorpora & 151 & 47,5 \\
\hline No incorpora bibliografía & 3 & ,9 \\
\hline Total & 318 & 100,0 \\
\hline
\end{tabular}

Los materiales que abundan en las bibliografías de los programas de los cursos tienen autoría masculina, ello sin ahondar en que este tipo de material documental puede afianzar propuestas clásicas de un sistema mundo patriarcal y androcéntrico, que tiende a opacar los aportes de la teoría feminista y sus consecuentes valoraciones como enfoque o perspectiva de género (tabla 4).

Para dar validez o contenido a dicha valoración recordemos lo que ya Marta Lamas (2006) nos decía al referirse a que la teoría feminista y la discusión teórica ha permitido descifrar las determinaciones situacionales $\mathrm{y}$ relacionales de las personas. Así bien, las teorizaciones y análisis, asumiendo un enfoque de género, nos permite la ampliación de la mirada "para ahondar en nuevas explicaciones sobre consecuencias discriminatorias de

Recuadro 2: Según estudios realizados cerca de $55 \%$ de los artículos en revistas electrónicas de corte educativo son escritos por mujeres (Fainholc, 2011), pero esto no se evidencia en la incorporación de ese material bibliográfico en los planes de estudio y programas en particular. la simbolización sexual" (p.119) que también se ha encarnado en la forma en la que conocemos y en quién recae o se corporaliza el conocimiento. Ver recuadro 2. 
Así, el identificar que aunque esta ampliación de la mirada nos permite ubicar la presencia del aporte de las mujeres de la academia aún hoy en nuestra casa de enseñanza y particularmente en la malla curricular de las diferentes disciplinas de estudio, no se tiene una presencia paritaria, ni siquiera, simétrica en relación con su lugar en cuanto a los aportes al conocimiento de las Ciencias Sociales. Por ello es innegable la necesidad de repreguntarnos ¿por qué revisar la educación formal desde la perspectiva de género? ¿Quiénes y cómo se establecen los criterios para definir como válida una producción intelectual? ¿Por qué considerar como relevante quién escribe y quién se presenta como escritor $u$ autor válido para que sea digno de ser presentado y leído por estudiantes que cursan las diferentes carreras en los planes de estudio de la Facultad? Observemos la información contenida en el tabla 5.

Tabla 5

Presencia de autoras por asignación de cursos según sexo. Programas de la Facultad de Ciencias Sociales $n=318$.

\begin{tabular}{lrccc}
\hline $\begin{array}{l}\text { Presencia de } \\
\text { Autoras }\end{array}$ & $\begin{array}{c}\text { Asignación de cursos según sexo } \\
\text { Valores relativos }(\%)\end{array}$ \\
\hline & $\begin{array}{c}\text { Mujer } \\
(\mathrm{n}=120)\end{array}$ & $\begin{array}{c}\text { Hombre } \\
(\mathrm{n}=156)\end{array}$ & $\begin{array}{c}\text { No indica } \\
\text { asignación } \\
(\mathrm{n}=24)\end{array}$ & $\begin{array}{c}\text { Se asigna a una } \\
\text { mujer y a un hombre } \\
(\mathrm{n}=18)\end{array}$ \\
$\begin{array}{l}\text { Se incorpora } \\
\text { Se incorpora en parte }\end{array}$ & 34,2 & 10,9 & - & 27,8 \\
No se incorpora & 30,8 & 34,6 & 8,3 & 44,4 \\
$\begin{array}{l}\text { No incorpora } \\
\text { bibliografía }\end{array}$ & $, 54,2$ & 53,2 & 91,7 & 27,8 \\
\hline Total & $\mathbf{1 0 0 , 0}$ & $\mathbf{1 0 0 , 0}$ & $\mathbf{1 0 0 , 0}$ & $\mathbf{1 0 0 , 0}$ \\
\hline
\end{tabular}

Los cursos que presentan con mayor frecuencia la incorporación de material documental escrito por mujeres se pueden identificar en los programas donde la persona que lo facilita es una académica. Si realizamos una sumatoria entre una incorporación importante y parcial (entre 5 y 10 , y entre 1 y 4 documentos), obtenemos que más de $60 \%$ de los programas impartidos por mujeres presentan como constante la incorporación de referencias bibliográficas con aportes de mujeres escritoras. La no incorporación de material bibliográfico con presencia de autoras se evidencia más en los programas donde el titular es un académico. 
Como hemos descrito, se pudo identificar una tímida incorporación de material bibliográfico elaborado por mujeres, también podemos realizar inferencias con respecto a la poco o casi nula presencia de material de creación propia, tanto para el caso de los académicos como para las académicas que trabajan en las diferentes unidades académicas de la Facultad de Ciencias Sociales.

La incorporación de material de autoría propia en los cursos que se imparten no es una práctica muy habitual ni en los hombres ni en las mujeres $(85 \%)$ profesionales en el área de las Ciencias Sociales.

En general del total de los programas analizados podemos visibiliza que aunque los hombres tienden a incorporar material de autoría propia en los cursos que imparten, los porcentajes con respecto a la no evidencia de incorporación de este tipo de material supera el 80\% de esa valoración (Ver figura 3).

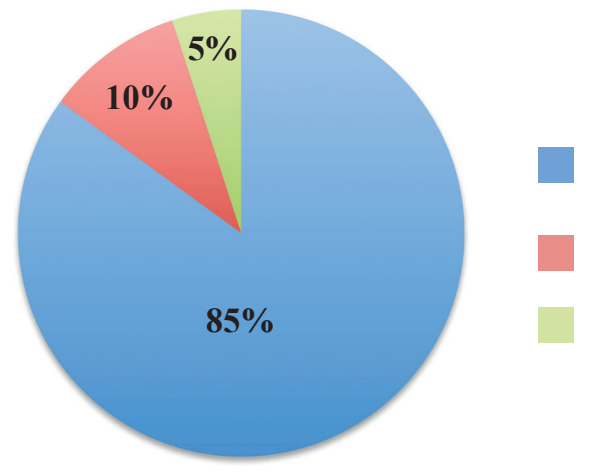

No se evidencia material de elaboración propia

Elaborado por varones

Elaborado por mujeres

Figura 3. Artículos y otras experiencias académicas de elaboración propia. Facultad de Ciencias Sociales n=318.

\section{Uso de lenguaje inclusivo}

En relación con el uso de lenguaje inclusivo aún se proyectan avances muy incipientes. Por ejemplo, se hace uso de la incorporación de los artículos la/lo, pero no se expone de manera idónea con las palabras o discursos que les preceden de manera inclusiva. En correspondencia los programas asignados a mujeres presentan una tendencia a la utilización o inclusión de este aspecto con mayor constancia y si realizamos una sumatoria entre los porcentajes que se obtiene en las categorías de "se evidencia" y se "evidencia en parte" - el uso de lenguaje inclusivo-, tendríamos que 57,5\% de los programas de los cursos en los que se registra a una profesora impartiéndolos, hay intentos por incorporar lenguaje inclusivo en sus programas. 
Los programas presentados por profesores registran una tendencia a la "no incorporación" de lo que podríamos considerar, como un tipo de acción afirmativa, en relación con la transversalización de la perspectiva de género (tabla 6).

Tabla 6

Uso de lenguaje inclusivo según persona que facilita el curso. Facultad de Ciencias Sociales $n=318$

\begin{tabular}{lcccc}
\hline $\begin{array}{l}\text { Usos de lenguaje } \\
\text { inclusivo }\end{array}$ & \multicolumn{5}{c}{$\begin{array}{r}\text { Asignación de cursos según sexo } \\
\text { Valores relativos }(\%)\end{array}$} \\
\cline { 2 - 5 } & $\begin{array}{c}\text { Mujer } \\
(\mathrm{n}=120)\end{array}$ & $\begin{array}{c}\text { Hombre } \\
(\mathrm{n}=156)\end{array}$ & $\begin{array}{c}\text { No indica } \\
\text { asignación } \\
(\mathrm{n}=24)\end{array}$ & $\begin{array}{c}\text { Se asigna a una } \\
\text { mujer y a un } \\
\text { hombre }(\mathrm{n}=18)\end{array}$ \\
\hline Se evidencia & 25,8 & 14,7 & - & 16,7 \\
Se evidencia en parte & 31,7 & 19,9 & - & 16,7 \\
No se evidencia & 42,5 & 65,4 & - & 66,7 \\
\hline Total & $\mathbf{1 0 0 , 0}$ & $\mathbf{1 0 0 , 0}$ & $\mathbf{1 0 0 , 0}$ & $\mathbf{1 0 0 , 0}$ \\
\hline
\end{tabular}

Es claro, desde la apropiación de un conocimiento situado y desde una perspectiva crítica, identificar que el lenguaje construye, crea, valida, y posiciona o ha posicionado afirmaciones que no necesariamente se corresponden con la realidad, por ejemplo: homogeneizar bajo el término hombre la diversidad humana. También, el lenguaje como vehículo de comunicación, expresa realidades que valoran como genuino o natural, prácticas de discriminación con esquemas o estructuras hegemónicas enajenantes que no favorecen o niegan la existencia de la mitad de la población (Fainholc, 2011).

\section{Estrategias pedagógica inclusivas}

Como parte de los procesos de transversalización de la perspectiva de género, nos abocamos a identificar si en los programas de los cursos que se imparten en la Facultad se registran algunas de las experiencias o particularidades de las mujeres y otros segmentos poblacionales que han sido vulnerados históricamente.

La mirada investigativa desde la perspectiva de género nos habla de una "política de la presencia" que supone que una auto-representación de las mujeres y sus particularidades no desde una posición esencialista o una idealización lo que se ha construido en términos androcéntricos como ser mujer, sino como el reconocimiento de procesos sociohistóricos de 
discriminación, permitiendo así, espacios de y para la discusión política, donde se valore en términos institucionales sus intereses, sus necesidades y la lucha por defender y asegurar satisfactores que les permitan un desarrollo autónomo y en equidad libre de todo tipo de violencias.

Por ello cuando se piensa en "particularidades de las mujeres" o algún tipo de acción afirmativa tanto para mujeres como varones que asisten a las clases nos referimos aquí a unos elementos básicos con respecto a potenciación de capacidades y planteamientos referidos con reivindicaciones de género; recordemos que las acciones afirmativas surgen como un intento por paliar condiciones de desigualdad con una tendencia a reducir actuaciones injustas y que están dirigidas a privilegiar a determinados grupos que en términos histórico-culturales han sido discriminados (Beltrán, 2008), por ello en este caso las acciones afirmativas podrían recaer principalmente en las mujeres. En el caso del análisis que se coloca aquí podemos observar que la incorporación de la política de la presencia y algunas valoraciones referidas a una acción afirmativa es mínima.

Es necesario preguntarnos cómo podemos, académicos y académicas, hacer efectivos postulados y valoraciones éticas con respecto al fomento de las diversidades y los derechos de las personas cuando los procesos históricos de invisibilización y negación de las condiciones de desigualdad han minado los espacios y escenarios donde transitamos, tanto en el marco institucional y el recorrido de la educación formal e informal. Nos queda mucho por recorrer en el reconocimiento de las necesidades de quienes han sido y son simbólica y materialmente invisibilizadas, negadas.

Los programas de la Facultad analizados no evidencian acciones afirmativas (68\%), ni se visibiliza en términos positivos la presencia de mujeres en alguna parte del programa $(86,6 \%)$ para las personas que participan como estudiantes en los diferentes cursos de las carreras en la Facultad de Ciencias Sociales. Veamos las siguientes valoraciones que se presentan en el tabla 7 y que dan cuenta de los limitados avances en materia de transversalización de la perspectiva de género en la malla curricular de las diferentes áreas disciplinarias de nuestra Facultad de Ciencias Sociales.

Es además relevante mencionar que en la "narrativa" o discurso presentado en los programas se evidencia casi una anulación o limitada importancia hacia las personas que participan en los cursos. Lo que evidencia de manera primordial el programa es la temática particular de cada disciplina. Esa no evidencia, está presente en más de la mitad de los programas analizados (ver figura 4) y no hay diferencias estadísticamente significativas con respecto a si quien se registra como titular o persona que facilita el curso es una profesora o un profesor. 
Tabla 7

Visibilización de las mujeres en alguna parte del Programa de Curso. Facultad de Ciencias Sociales $\boldsymbol{n}=\mathbf{3 1 8}$

\begin{tabular}{|c|c|c|c|c|}
\hline \multirow[t]{2}{*}{$\begin{array}{l}\text { Particularidades de las mujeres } \\
\text { en los programas }\end{array}$} & \multicolumn{3}{|c|}{$\begin{array}{c}\text { Valores relativos } \\
(\%)\end{array}$} & \multirow[t]{2}{*}{ Total } \\
\hline & $\begin{array}{c}\text { Se } \\
\text { evidencia }\end{array}$ & $\begin{array}{l}\text { Se evidencia } \\
\text { en parte }\end{array}$ & $\begin{array}{l}\text { No se } \\
\text { evidencia }\end{array}$ & \\
\hline $\begin{array}{l}\text { ¿Se visibiliza la presencia de las mu- } \\
\text { jeres en alguna parte del programa? }\end{array}$ & 14,5 & 17,3 & 68,2 & 100,0 \\
\hline $\begin{array}{l}\text { ¿Se registra o percibe la presencia } \\
\text { de algún tipo de acción afirmativa } \\
\text { en el programa? }\end{array}$ & 3,8 & 9,7 & 86,5 & 100,0 \\
\hline $\begin{array}{l}\text { ¿Se visibilizan actividades peda- } \\
\text { gógicas o didácticas que incluyan } \\
\text { los derechos de las personas? }\end{array}$ & 5,7 & 15,7 & 78,6 & 100,0 \\
\hline $\begin{array}{l}\text { ¿Se presenta trabajo de campo } \\
\text { o extra aula donde considere las } \\
\text { particularidades de las mujeres? }\end{array}$ & 4,1 & 11,0 & 84,9 & 100,0 \\
\hline
\end{tabular}

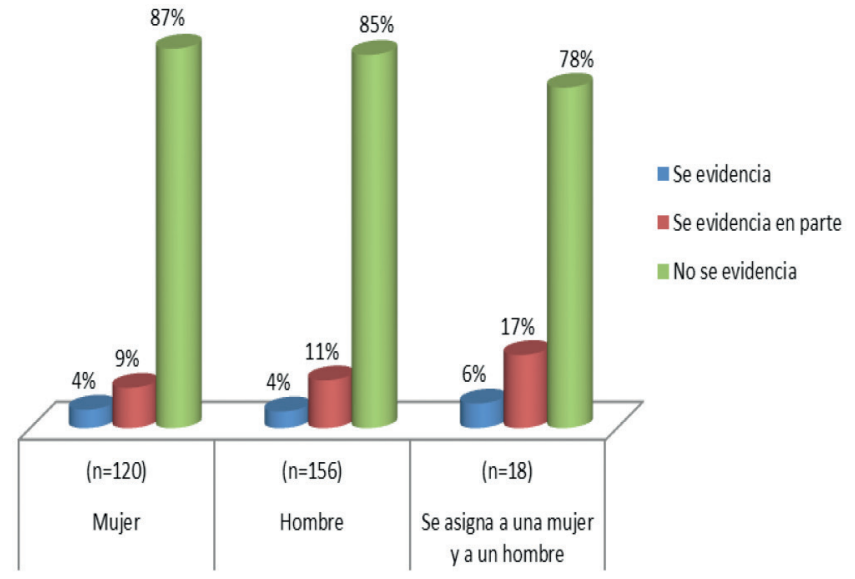

Figura 4. Presencia de acciones afirmativas según persona que facilita el curso Facultad de Ciencias Sociales.

Mucho se ha planteado sobre los procesos de enseñanza-aprendizaje y las diversas formas en las que las personas aprehendemos; sin embargo aquí también obtenemos una calificación regular, cuando se trata de facilitar 
procesos de enseñanza género-sensitivos y respetuosos de los saberes que tienen tanto personas facilitadoras como personas que participan de la formación, en cualquiera de las áreas en la que se esté potenciando sus habilidades y capacidades.

Estas valoraciones también las podemos relacionar con la forma tradicional en la que se imparten las clases, la misma frase de "impartir cátedra", "dictar la lección", "impartir ciencia", nos puede da pistas sobre lo estanco $\mathrm{y}$ unidireccional que puede ser la forma en la que trasmite el conocimiento disciplinar. El estudiantado se sienta en el banquillo de la clase y en el mejor de los casos tiene al frente a un profesor o profesora que imparte un conocimiento que cada quien debe internalizar para "ganar" un curso. La modalidad de clases magistrales aún sigue siendo importante y se visibiliza en más del treinta por ciento de los programas de los cursos que se han analizado (Ver figura 5).
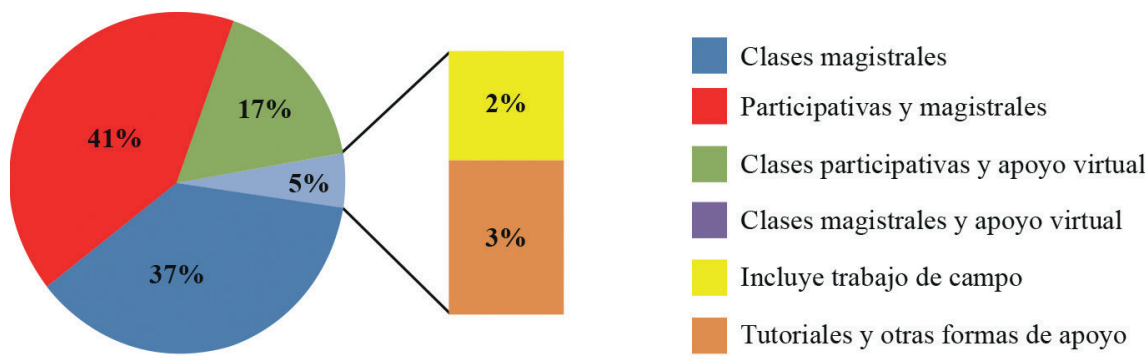

Figura 5. Estrategia metodológica, pedagógica que se registra en el programa $\mathrm{n}=318$

Las estrategias que se indican en los programas como parte de la estrategia metodológica del curso aunque muestran una importante presencia en relación con el desarrollo de clases participativas combinadas con la forma tradicional o lo que conocemos como clases magistrales (40\%), nos invitan a valorar a qué le llamamos participativo. ¿Es participativo solicitar que estudiantes "nos expongan" o presenten las lecturas asignadas para el estudio, es ésta, una propuesta pedagógica participativa-inclusiva? ¿Será que aún prevalece una forma jerárquica y lineal de asumir el proceso formativo? Esto es precisamente lo que se evidencia desde nuestro proceso investigativo.

\section{¿Nuestros hallazgos, tareas de la Facultad de Ciencias Sociales de la Universidad Nacional de Costa Rica?}

En relación con el recorrido por los hallazgos de nuestra investigación, les compartimos las conclusiones a las que llegamos como equipo investigador: 
Asumimos que la perspectiva de género es una herramienta que enriquece formas diversas y complejas en la apropiación del conocimiento, siendo una perspectiva teórica transdisciplinar que se enriquece con la visibilización de referentes metodológicos abiertos hacia unas comprensiones complejas de la realidad social, la Facultad de Ciencias Sociales debe de sumir hoy un posicionamiento crítico que se aleje de una visión tradicional de la forma de construir ciencia, de forma tal que trascienda los límites de la igualdad y los derechos civiles. La enseñanza de las ciencias sociales se priva de las aportaciones de un enfoque género sensitivo abierto a la construcción de saberes.

En la Universidad Nacional contamos con un marco normativo en relación a la transversalidad de género en el quehacer académico-institucional; sin embargo, de acuerdo con lo que plantean los programas de cursos de las carreras de la Facultad de Ciencias Sociales, su aplicación efectiva en la formación profesional de las estudiantes y los estudiantes, es prácticamente ausente. Anotar el término género como un enunciado en el programa no constituye un proceso de transversalización.

La Facultad de Ciencias Sociales continua sosteniendo una deuda histórica considerable con respecto a la equidad de género, porque no cuenta con el mínimo de paridad entre el personal académico que tiene cursos asignados, $67 \%$ son hombres y $33 \%$ son mujeres.

El material bibliográfico de autoría masculina, sigue predominando en la producción del conocimiento, siendo referente teórico para los procesos de enseñanza-aprendizaje. Ello incide en la no utilización de material escrito por mujeres, invisivilizando su lugar en la construcción de la ciencia.

A partir del estudio de las unidades de análisis de nuestra investigación -programas de curso- nos hemos cuestionado de forma significativa el efecto de invisibilización de la autoría en la producción intelectual, que impacta tanto a los hombres como a las mujeres. Sin embargo, este efecto, tiene consecuencias negativas, en mayor grado para las mujeres académicas/intelectuales, quienes históricamente han sido anuladas como generadoras de conocimientos y creadoras de ciencia, por lo tanto, el formato de la American Phychological Association (APA), para referenciar autorías, se convierte en un elemento que colabora en la perpetuación de la invisibilización de las mujeres en la academia. Ante esto, consideramos imperativa la reflexión y el uso de este tipo de normativas que validan la producción intelectual del conocimiento desde una visión patriarcal.

Con respecto a las estrategias pedagógicas, se evidencia en los programas de los cursos que las clases magistrales siguen siendo la estrategia metodológica 
de enseñanza-aprendizaje más favorecida por el personal docente de la Facultad, aunque se visualiza un importante esfuerzo por incluir la participación en clase como parte del proceso formativo, queda un cuestionamiento ineludible ¿qué estamos entendiendo y aplicando como metodologías participativas?

En cuanto al lenguaje inclusivo, su empleo en los programas es sumamente ligero. Es posible evidenciar una tendencia diferenciada entre profesores y profesoras, siendo en los programas de las mujeres en los que se ubica mayor presencia y mejor empleo de lenguaje inclusivo no sexista.

Es necesario desarrollar y fortalecer las capacidades institucionales y humanas para poner en ejecución estrategias y acciones que faciliten la incorporación del enfoque de género en términos teórico-metodológicos para la formación y en términos género-sensitivos para potenciar relaciones equitativas libres de discriminación.

\section{Referencias}

Beltrán, E. (2008). Justicia, democracia y ciudadanía: Las vías hacia la igualdad. En E. Beltrán y V. Malquiera (Eds.), Feminismos. Debates teóricos contemporáneos (pp. 191-242). Madrid: Alianza Editorial.

Fainholc, B. (2011). Educación y género: una perspectiva, social, cultural, y tecnológica. Buenos Aires: Lugar Editorial.

FLACSO. (2006). Guía para la transversalización de género en el PNUD. Chile: PNUD.

Haraway, D. J. (1995). Ciencia, cyborgs y mujeres. La reinvención de la naturaleza. Madrid: Ediciones Cátedra.

Lagarde, M. (1996). Los cautiverios de las mujeres: madresposas, monjas, putas, presas y locas. México: UNAM.

Lamas, M. (2006). Feminismo. Transmisiones y Retransmisiones. México: Taurus.

Monzón, A. (2009). Mujeres, ciencia e investigación, miradas críticas. Guatemala: Universidad de San Carlos.

Monzón, A. (2011). Mujeres Escribas: Tejedoras de pensamiento. Guatemala: FLACSO.

UNA. (2010). Política de Igualdad y Equidad de Género. Heredia: GACETA No 09-2010, 15 de junio.

Universidad Nacional (2014). Lista de cursos del 2014, Bachillerato y Licenciatura de las carreras de la Facultad de Ciencias Sociales. HePNUD (s. f.). Marco Estratégico Regional de Género del PNUD en América Latina y Caribe 2005-2009. Chile: PNUD. 